\title{
Sun, Earth, radioactive ore: Common periodicity
}

\author{
O. B. Khavroshkin, V. V. Tsyplakov \\ Institute of Physics of the Earth, RAS, Moscow, Russia; khavole@ifz.ru
}

Received 14 June 2013; revised 14 July 2013; accepted 21 July 2013

Copyright (C) 2013 O. B. Khavroshkin, V. V. Tsyplakov. This is an open access article distributed under the Creative Commons Attribution License, which permits unrestricted use, distribution, and reproduction in any medium, provided the original work is properly cited.

\section{ABSTRACT}

The study of natural radioactivity of objects which are able to change their temporal timing feature is presented. It is of interest to compare the latest data on the activity of the Sun and the periodicity of solar neutrinos and the temporal characteristics of the radioactive source. That is, to conduct a search for the possible influence of external sources for radioactivity. There are cycles $5 \mathrm{~min}, 18 \mathrm{~min}$ and $53 \mathrm{~min}$ found in solar physics. The cycle of 27 days corresponds to the activity of the Sun. During of the solar activeity these temporal pulsations are lost in a strong variation of solar wind (Neugebauer, NASA). The Stanford University scientists (P. Starrek, G. Valter and $M$. Vitlend) have found the cycle of $\mathbf{2 8 . 4}$ days as pulsations of the solar neutrinos. Neutrinos come from the depths of the Sun and they tell about the frequency of oscillations of solar bowels. It is also seen online: Kostyantynivska $L$. V. Solar activity. Search experiment is better to have a known but modified experiment. Experiments on monitoring natural radioactivity and the possible influence from the Sun were previously carried out by measuring the variations of the gamma-ray sample of ore from the TransBaikal uranium deposit; the characteristics of the sample are known. The spectrum of temporal variations in the activity of the sample Zabaikalskaya radioactive ore contains peaks which coincide with the period of natural oscillations of the Sun. The capture cross section of the radioactive heavy deformed nucleus in time decay increases in many orders and is able to interact with the stream of solar neutrinos which are modulated by own oscillations of the Sun. The picks of spectrum of long-period oscillations of the Earth exceed its own contain peaks that match the value with an accuracy of $1 \%-3 \%$ with peaks of its own oscillations of the Sun.
The mechanism of excitation of these oscillations is similar to the nature of variations in the activity of a radioactive sample of ore. These effects are included in the mechanisms of interaction of the Earth-the Sun systems and impact on seismicity; search problem of existing natural nuclear reactor inside Earth core.

Keywords: Solar R-Index; Radioactive Source; Periodicity of Radioactivity; Solar Neutrino Oscillations; Super-Long Oscillations of the Earth; Abnormal Capture Cross Section of Neutrino

\section{INTRODUCTION}

The study of natural radioactivity of objects which are able to change their temporal timing feature is presented [1]. It is of interest to compare the latest data on the activity of the Sun and the periodicity of solar neutrinos and the temporal characteristics of the radioactive source. That is, to conduct a search for the possible influence of external sources for radioactivity. There are cycles $5 \mathrm{~min}$, $18 \mathrm{~min}$ and $53 \mathrm{~min}$ found in solar physics. The cycle of 27 days corresponds to the activity of the Sun. During of the solar activity these temporal pulsations are lost in a strong variation of solar wind (Neugebauer, NASA). The Stanford University scientists (P. Starrek, G. Valter and M. Vitlend) have found the cycle of 28.4 days as pulsations of the solar neutrinos. Neutrinos come from the depths of the Sun and they tell about the frequency of oscillations of solar bowels. It's also seen online: Kostyantynivska L. V. Solar activity. Search experiment is better to have a known but modified experiment.

\section{RESEARCH AND PRIMARY DATA PROCESSING}

Experiments on monitoring natural radioactivity and the possible influence from the Sun as previously carried out by measuring the variations of the gamma-ray sample of ore from the Trans-Baikal uranium deposit; the 
characteristics of the sample are known [1]. Used standalone DVR "E Clerk" with every minute polling detection device (radiometer) and then transfer the data to a computer over a simple diagram (Figure 1).

Accordingly sealed steel container volume of 0.5 litre which containing $20 \mathrm{~g}$. uranium ore 1) as the source is permeable only for gamma-rays which are recorded radiation detector (Geiger counter type SBM19), and 2) the resulting level of radiation was written to memory every minute stand-alone digital recorder "E Clerk". 3) These data are then processed on a personal computer. 4) Ore sample was placed in a sealed steel container for limiting the influence of atmospheric pressure, virtually pressure variations in ore no effect (meaning the amount of radon as a decay product) and passed through the wall of the container only gamma radiation and variations of betaradiation is not registered. Figure 2 shows an example of recording variations of gamma radiation averaged over the 3rd minute, recorded from 1 to 18 March 2013.

These records must be compared with the existing state of the Sun. Since the initially criterion to allocate the current state of the Sun is difficult preference was given to the $\mathrm{K}_{\mathrm{p}}$-index as the most common. Therefore, during the same time period of observation of gammaradiation was built a series of numbers of $\mathrm{K}_{\mathrm{p}}$-index (Figure 3(a)). To compare the parallel series of records gamma-radiation and $\mathrm{K}_{\mathrm{p}}$-index synchronous variations of the ore sample averaged gamma radiation on the 3 hour intervals (Figure 3(b)). After that there is determined by the correlation function of a sliding 60 hour window (20 independents points; Figure 3(c)).

When assessing the value of the correlation coefficients obtained in window $60 \mathrm{hr}(n=20$ independent points) in $t$-Fisher criterion

$$
t=r \times \sqrt{n-2} / \sqrt{1-r^{2}}
$$

for the obtained correlation coefficient $r=-0.733$ on the Student's table $t$-distribution we received double acting significance on level of 0.001 , that is with probability $\mathrm{P}>$ 0.999 there is a relationship between the variations of gamma-ray ore and $\mathrm{K}_{\mathrm{p}}$-index, which proves the existence of a link between solar processes and variations of gamma-ray of the ore. Next was obtained spectrum of

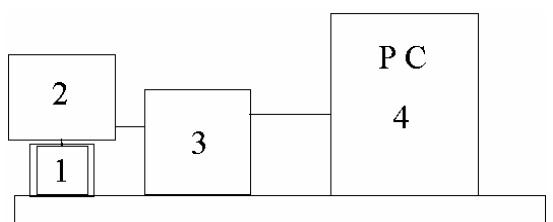

Figure 1. Scheme of registration of variations of gamma radiation sample. 1) container, 2) radiation detector, 3) DVR, 4) a personal computer.

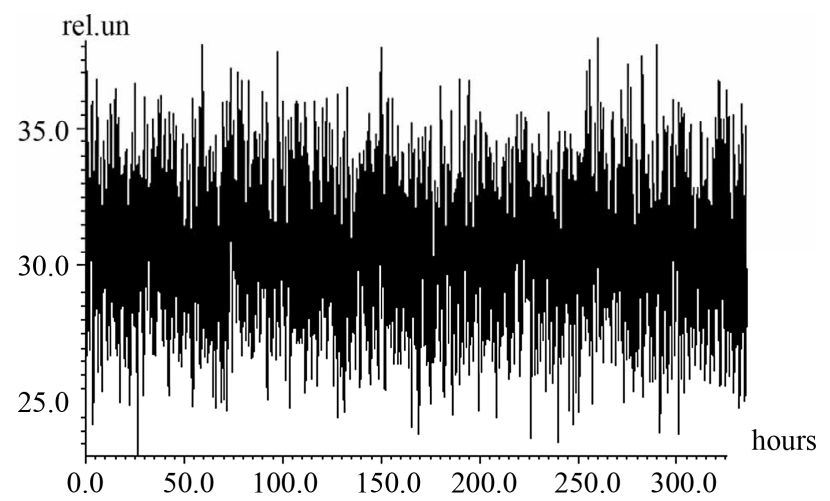

Figure 2. Example of record variations of gamma-radiation of ore at 3-minute average.

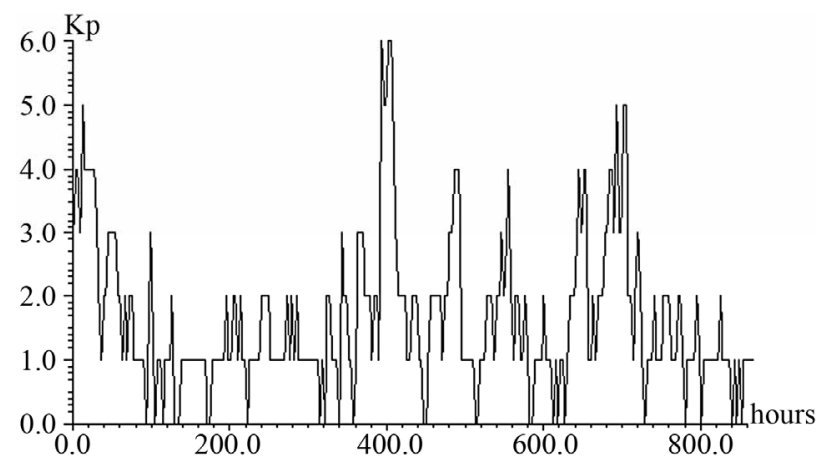

(a)

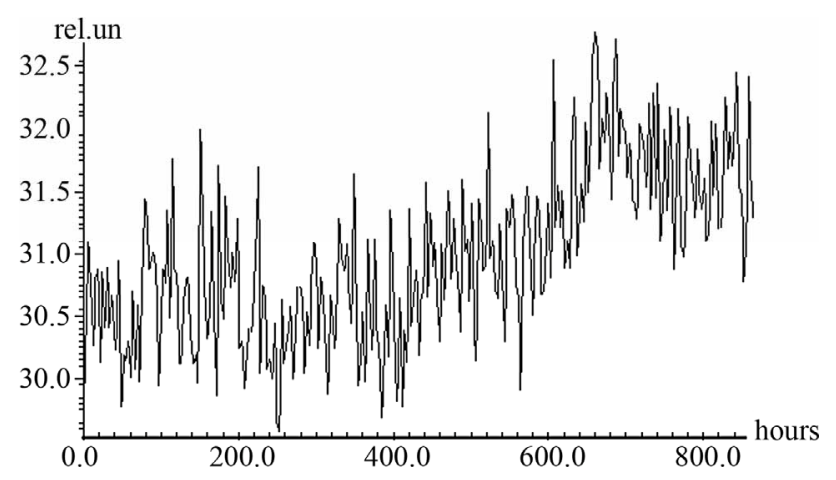

(b)

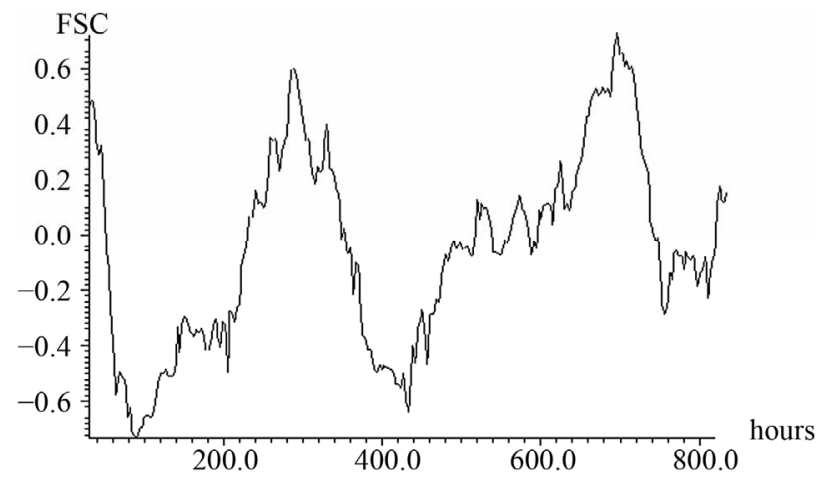

(c)

Figure 3. Temporal variations of solar $\mathrm{K}_{\mathrm{p}}$-index (a) and gamma-ray radiation of ore (b), and feature a sliding correlation between them (a, b) in March 2013. 
variations of gamma radiation the ore for all of March at 3-minute averaging (Figure 4). Even a simple analysis of this spectrum indicated the existence of spectral peaks coinciding with the period of natural oscillations of the Sun, as well as the existence of a daily component. Regarding the periodicity of radon, unfortunately do whatany conclusions impossible - too noisy channel by much exposure to radon flux processes.

Joint analysis of the spectral data of the temporal variations gamma radiation the ore and natural oscillations of the Sun are given in Table 1. Data on the theoretical values of periods of natural oscillations of the Sun are presented below (Table 2) [2].

A comparison of Tables 1 and 2 and the data in Table 1 should be plural coincidence of periodicities in the time variations of the activity of a radioactive source with the frequency of the oscillations of the Sun's own up to 3, 4 digits. The very existence of diurnal components also proves relationship with solar processes as the Earth can shield the sun's rays or particles that affect the variation of gamma-ray ore. The presence of a significant negative correlation in the chart FSK (Figure 3(c)) with low K index indicates depth of wave processes on the Sun affect the variation of gamma-ray ore.

\section{THERE IS ABOUT THE PHYSICAL MECHANISMS OF THE SUN OSCILLATIONS INFLUENCE ON THE ORE SAMPLE RADIOACTIVITY}

Since the intensity of the radiation neutrino is modulated by solar oscillations presumably, the problem of interpretation lies in the small capture cross section [3]. The scattering cross section of solar neutrinos is about $10^{-21}-10^{-18}$ barn, depending on the energy of the neutrino. For the container (Figure 1) the number $N$ of neutrinos per unit time experienced the interaction with nuclei provided single interaction of each particle (thin target) is given by

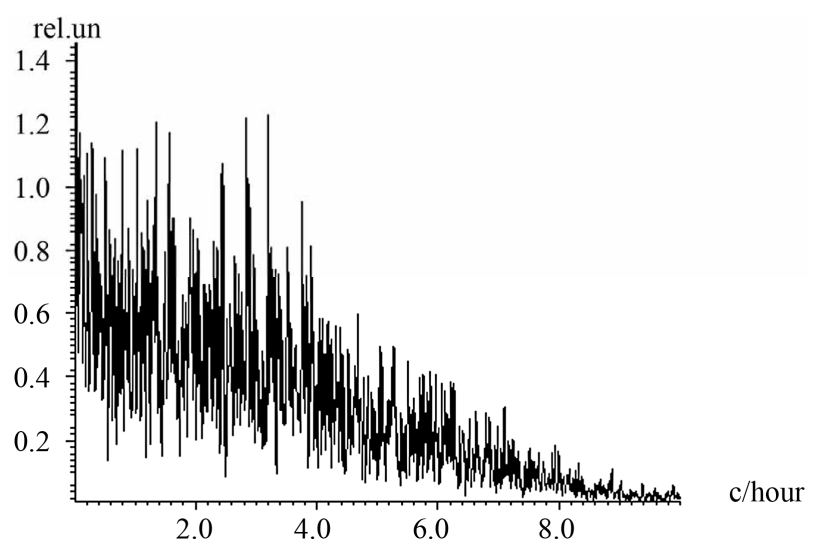

Figure 4. An example of the spectrum of temporal variations of gamma radiation the ore sample in March 2013.
Table 1. Significant $(P>0.95)$ of the observed variations in the periods of the ore gamma - ray and for comparison - natural periods oscillations of the Sun.

\begin{tabular}{|c|c|c|c|}
\hline $\mathrm{N}$ & $\begin{array}{c}T_{r} \text { (hour, min.) } \\
\text { observed }\end{array}$ & $\begin{array}{c}T_{s}(\min .) \\
\text { (Theoretical) }\end{array}$ & $\begin{array}{c}\text { Note } \\
\text { (Mode) }\end{array}$ \\
\hline 2 & $6.4 \mathrm{~h}$ & - & - \\
\hline 1 & $24.0 \mathrm{~h}$ & - & Days \\
\hline 3 & $166.2 \mathrm{~m}$ & $166.7 \mathrm{~m}$ & $g 15, l 3$ \\
\hline 4 & 115.9 & 118.9 & $g 10 . l 3$ \\
\hline 5 & 75.9 & $74.9 ; 76.8$ & $g 5, l 3 ; g 7, l 4$ \\
\hline 6 & 62.05 & 62.29 & $p 1, l 1$ \\
\hline 7 & 58.0 & $57.25 ; 57.73$ & $p 1, l 2 ; g 3, l 4$ \\
\hline 8 & 44.8 & 44.18 & $g 1, l 4$ \\
\hline 9 & 36.8 & 36.98 & $p 2, l 1$ \\
\hline 10 & 31.7 & 32.19 & $p 2, l 2$ \\
\hline 11 & 25.6 & 25.09 & $p 3, l 2$ \\
\hline 12 & 24.7 & 24.49 & $p 4, l 0$ \\
\hline 13 & 20.8 & 20.52 & $p 4, l 2$ \\
\hline 14 & 18.8 & 18.68 & $p 4, l 1$ \\
\hline 15 & 15.9 & 15.72 & $p 5,14$ \\
\hline 16 & 15.6 & 15.72 & $p 5, l 4$ \\
\hline 17 & 14.8 & 14.93 & $p 7, l 0$ \\
\hline 18 & 14.2 & 14.08 & $p 7, l 1$ \\
\hline 19 & 13.6 & $13.81 ; 13.35$ & $p 6, l 4 ; p 7, l 2$ \\
\hline 20 & 12.96 & 12.77 & $p 7,13$ \\
\hline 21 & 11.36 & 11.34 & $p 9, l 1$ \\
\hline 22 & 10.73 & 10.78 & $p 10, l 0$ \\
\hline 23 & 10.41 & 10.49 & $p 9, l 3$ \\
\hline 24 & 10.3 & 10,35 & $p 10, l 2$ \\
\hline 25 & 10.17 & 10.18 & $p 9, l 4$ \\
\hline 26 & 9.6 & $9.65 ; 9.54$ & $p 10, l 3 ; p 11, l 2$ \\
\hline 27 & 9.05 & 9.15 & $p 12, l 0$ \\
\hline 28 & 8.85 & 8.84 & $p 12, l 1$ \\
\hline 29 & 8.75 & 8.71 & $p 11, l 4$ \\
\hline 30 & 8.59 & 8.56 & $p 12, l 2$ \\
\hline 31 & 8.45 & 8.50 & $p 13, l 0$ \\
\hline 32 & 8.33 & 8.32 & $p 12, l 3$ \\
\hline 33 & 7.98 & 7.99 & $p 13, l 3$ \\
\hline 34 & 7.61 & 7.60 & $p 13, l 4$ \\
\hline 35 & 7.51 & 7.49 & $p 14, l 2$ \\
\hline 36 & 7.4 & 7.45 & $p 14, l 0$ \\
\hline 37 & 7.2 & 7.25 & $p 15, l 1$ \\
\hline 38 & 6.93 & 6.89 & $p 15, l 3$ \\
\hline 39 & 6.76 & 6.75 & $p 15, l 4$ \\
\hline
\end{tabular}

On Table 1: $T_{r}$ - frequency radiation of the radioactive source; $T_{s}$-natural period of oscillation of the Sun; $g$, $p$-mode of natural oscillations of the Sun.

$$
N=j n S 1 \sigma=j M \sigma
$$

where $j$-particle flux density, $\sigma$ - the effective cross section of scattering particles nucleus; $n$-number of nuclei per unit volume of the container $\left(\right.$ in $\mathrm{cm}^{-3}$ ); $S$-irradiated target area (in $\left.\mathrm{cm}^{2}\right) ; 1$ - target thickness (in $\mathrm{cm}$ ); 
Table 2. The periods of solar oscillations for Sun standard model [2].

\begin{tabular}{|c|c|c|c|c|c|}
\hline \multirow{2}{*}{ Mode } & \multicolumn{5}{|c|}{ Period, min. } \\
\hline & $l=0$ & $l=1$ & $l=2$ & $l=3$ & $l=4$ \\
\hline$p 1$ & 62.29 & 57.25 & 42.50 & 39.53 & 37.58 \\
\hline$p 2$ & 40.94 & 36.9B & 32.19 & 29.42 & 27.62 \\
\hline$p 3$ & 30.93 & 27.88 & 25.09 & 23.21 & 21.92 \\
\hline$p 4$ & 24.49 & 22.30 & 20.52 & 19.26 & 18.31 \\
\hline$p 5$ & 20.19 & 18.68 & 17.39 & 16.44 & 15.72 \\
\hline$p 6$ & 17.17 & 16.04 & 1540 & 14.38 & 13.81 \\
\hline$p 7$ & 14.98 & 14.08 & 13.35 & 12.77 & 12.32 \\
\hline$p 8$ & 13.21 & 12.55 & 11.97 & 11.51 & 1144 \\
\hline$p 9$ & 14.86 & 11.34 & 10.87 & 10.49 & 1048 \\
\hline$p 10$ & 10.78 & 10.35 & 9.97 & 9.65 & 9.39 \\
\hline$p 11$ & 9.90 & 9.54 & 9.21 & 8.94 & 8.71 \\
\hline$p 12$ & 9.15 & 8.84 & 8.56 & 8.32 & 841 \\
\hline$p 13$ & 8.50 & 8.23 & 7.99 & 7.78 & 7.60 \\
\hline$p 14$ & 7.94 & 7.71 & 7.49 & 7.31 & 7.15 \\
\hline$p 15$ & 7.45 & 7.25 & 7.06 & 6.89 & 6.75 \\
\hline$p 16$ & 7.02 & 6.84 & 6.67 & 6.52 & 6.39 \\
\hline$p 17$ & 6.64 & 6.47 & 6.32 & 6.18 & 6.06 \\
\hline$p 18$ & 6.29 & 6.14 & 6.00 & 5.87 & 5.77 \\
\hline$p 19$ & 5.98 & 5.84 & 5.71 & 5.60 & 5.50 \\
\hline$p 20$ & 5.69 & 5.56 & 5.45 & 5.34 & 5.25 \\
\hline
\end{tabular}

\begin{tabular}{ccccc}
\hline \multirow{2}{*}{ Mode } & \multicolumn{4}{c}{ Period, min } \\
\cline { 2 - 5 } & $l=1$ & $l=2$ & $l=3$ & $l=4$ \\
\hline$f$ & & 45.90 & 40.97 & 38.82 \\
$g 1$ & 61.58 & 55.05 & 47.94 & 44.18 \\
$g 2$ & 84.4 & 63.03 & 54.88 & 49.59 \\
$g 3$ & 105.3 & 72.58 & 61.88 & 57.73 \\
$g 4$ & 127.3 & 83.49 & 67.76 & 61.11 \\
$g 5$ & 149.2 & 95.38 & 74.9 & 64.89 \\
$g 6$ & 171.4 & 107.7 & 83.4 & 70.30 \\
$g 7$ & & 120.2 & 91.8 & 76.83 \\
$g 8$ & & 132.9 & 100.7 & 83.62 \\
$g 9$ & & 145.9 & 109.7 & 90.56 \\
$g 10$ & & 158.9 & 118.9 & 97.62 \\
$g 11$ & & 172.1 & 128.1 & 104.5 \\
$g 12$ & & & 137.6 & 111.7 \\
$g 13$ & & & 147.0 & 118.9 \\
$g 14$ & & & 156.5 & 126.5 \\
$g 15$ & & & 166.7 & 133.3 \\
$g 16$ & & & 175.9 & 141.5 \\
$g 17$ & & & & 148.6 \\
$g 18$ & & & & 156.4 \\
$g 19$ & & & & 164.0 \\
$g 20$ & & & & \\
\hline
\end{tabular}

On Table 2: $p, g, f-$ natural vibration modes of the Sun; $1-$ form of natural oscillations.
$M-$ total number of nuclei in the irradiated portion of the target. If we put: $j \sim 10^{10}$ chast $/ \mathrm{sm}^{2} \cdot \mathrm{s}, \mathrm{M} \sim 10^{25}$ units, $\sigma \sim$ $10^{-45}-10^{-42} \mathrm{~cm}^{2}$ get

$$
N \approx 10^{-9}-10^{-6} \text { particles/s. }
$$

Assessment in accordance with the above formula gives only a small amount of probability of interaction and require a new concept of capture cross section while explaining the periodicity of the solar neutrino easy. Indeed as a result of nuclear reactions in the solar interior the electronic neutrino with a wide range of kinetic energies are produces. It is believed that the amount of neutrinos and their kinetic energy spectrum does not change when the sun passes through the plasma, but it can change the beat wave processes. The total flux of neutrinos from the Sun on the Earth's surface is approximately $6.6 \times$ $10^{10}$ particle $/ \mathrm{cm}^{2} \cdot \mathrm{s}$. Full neutrino "luminosity" of the Sun regardless of the particular nuclei fusion processes is approximately $2 \times 3.85 \times 10^{20} \mathrm{MW} / 26.7 \mathrm{MeV}$, that is about $1.8 \times 10^{38}$ neutrinos in $1 \mathrm{sec}$. Speeds individual nuclear reactions and corresponding neutrinos are highly dependent on temperature and other parameters of chemical composition primarily on the helium content. Therefore the wave processes in the solar interior, affecting the temperature of the medium and mixing helium modulate the stream of neutrinos. It is also known that as the neutrino energy is a linear increase in total cross section of neutrino-nucleon interaction. Section can grow linearly with the energy up to the geometrical dimensions of the nucleon $\left(\sim 10^{-26} \mathrm{~cm}^{2}\right)$. According to experimental data in the container with the radioactive ore must have a strong interaction that is the capture neutrinos exceed all conceivable standards. The adopted model is not possible (see above), but it is crucial to note-all models of interaction considering the interaction with the stable nuclei. So some idea of fundamentally new approach to the creation of a workable model must take into account that the capture cross section must be evaluated for radioactive, unstable nucleus. As you know for over-energy neutrinos have non-transparent material. And if you follow the classical mechanics, the energy of the neutrino interaction with the unstable excited nucleus is the sum of the neutrino energy and neutrons in the nucleus before decay condition. This is enough energy to the energy state of a neutrino interacting with a radioactive ore of container. On the other hand one can imagine that quarks decaying nuclei form does not matter how small clouds of virtual $\mathrm{W}$-and Z-bosons around them and the more denser medium capable of absorbing solar neutrino. Capture of the neutrino stream modulated by own oscillations of the Sun shows the hidden periodicity of its. In the decay of uranium-235 to barium $139+$ kripton $95+\mathrm{E}_{\mathrm{p}}$. interaction cross section increases as the ratio of $\mathrm{K}=\mathrm{E}_{\mathrm{p}} / 3 \mathrm{Kt} / 2$. Since $\mathrm{E}_{\mathrm{p}}=202.5 \mathrm{MeV}=3.24 \times 10^{-11} \mathrm{~J} .3 \mathrm{kT} / 2=6.21 \times 10^{-21} \mathrm{~J}$, 
we obtain $\mathrm{K}=0.52 \times 10^{10}$. That is the number of neutrinos interacting in the standard model with $\mathrm{U} 235(\mathrm{~N} \approx$ $10^{-9}-10^{-6}$ particles/s.) may be increased to once and for $\mathrm{N}_{\mathrm{u}} \approx 0.5 \times 10-10^{4}$ particles/s. Thus detected gamma radiation measured $\sim 10^{2}$ events per second.

\section{SOLAR NEUTRINOS, SEISMICITY AND THE EARTH'S HEAT FLOW (RADIOACTIVE COMPONENT)}

According to various estimates, the radioactive component of the heat flow of the Earth is about $80 \%$ of the total energy. This component is due to the existence of natural radioactive sources, primarily the isotopes of uranium, thorium, potassium, and others [4]. Heat flow of the Earth is $7 \times 10^{-2} \mathrm{~W} / \mathrm{cm}^{2}$ or $1 \times 10^{-6} \mathrm{cal} / \mathrm{cm}^{2} \mathrm{sec}$. and determines the energy of the Earth heat engine including seismicity. Activity or signs of activity create a constant background literally heterogeneous (1.5-5-fold difference in level, Table 3, [10]). Activity the ore is much higher but in general we can assume that the constant background given its geological volumes as well as the ore is subject to modulation periods of natural oscillations of the Sun. That is the heat flow as a smooth function is perturbed by the known solar periods. And if the laboratory is registered as a variation of the activity the global scale in the rock mass of the crust and mantle these variations should show up as extra-long seismic oscillations with periods of solar oscillations. First of all, this effect is observed and discovered in the spectra of the Earth's oscillations [4-12]. Reliable and accurate match with the above model the interaction of solar neutrinos and a heavy radioactive element of the Earth and the Earth's crust require the creation of a new mechanism of the dynamics of solar-terrestrial links. On the other hand, according to MANKO V.I. from the Ph. Inst. RAS it is a consequence of the uncertainty relation for the coordinate-pulse in quantum systems in thermal equilibrium [13]. Processes of decay and fusion in the light of the different quantum correlations over a potential barrier which suggests a new section for the capture of solar neutrinos.

\section{GENERAL CONCLUSIONS}

The spectrum of temporal variations in the activity of the sample Zabaikalskaya radioactive ore contains peaks which coincide with the period of natural oscillations of the Sun.

1) The capture cross section of the radioactive heavy deformed nucleus in time decay increases in many orders and is able to interact with the stream of solar neutrinos which are modulated by own oscillations of the Sun.

2) The picks of spectrum of long-period oscillations of the Earth exceed its own and contain peaks that match the value with an accuracy of $1 \%-3 \%$ with peaks of its own oscillations of the Sun. The mechanism of excitation of these oscillations is similar to the nature of variations in the activity of a radioactive sample of ore.

3) These effects are included in the mechanisms of interaction of the Earth - the Sun systems and impact on seismicity; search problem of existing natural nuclear reactor inside Earth core.

\section{REFERENCES}

[1] Khavroshkin, O. and Tsyplakov, V. (2011) Radioactivity of nuclei in a centrifugal force field. The Natural Science (NS), 3, 733-737. doi:10.4236/ns.2011.38097

[2] Iben Jr., I. and Mahaffy, J. (1976) On the sun's acoustical spectrum. Astrophysical Journal, 209, L39-L43. doi: $10.1086 / 182262$

[3] (2005) The particles and nuclei. Experiment. (Reaction cross section). Moscow State University Press, Moscow.

[4] Telford, V.M., Geldart, L.P., Sheriff, R.E. and Case, D.A. (1980) Applied geophysics. "NEDRA", Moscow, 501c. Cambridge University Press, Cambridge, London, New York, Melbourne.

[5] Bullen, K.E (1966) Introduction to theoretical seismology. Springer-Verlag, New York, 460C.

[6] Khavroshkin, O.B. (1999) Some problems of nonlinear seismology. M.Sc, UIPE Sciences, 301c.

[7] Nikolaev, A.V., Rykunov, L.N., Starovoit, O.E., Khavroshkin, O.B. and Tsyplakov, V.V. (1986) Non-linear features of the natural oscillations of the Earth. Academy of Sciences of the USSR, The DNC, The POI. Dynamic Processes in Discrete Geophysical Systems. Collected Book, Vladivostok, 138c.

[8] Linkov, E.M. and Tipisev, S.Y. (1986) Observation of long-period oscillations of the Earth seismograph with a photoelectric transducer. Academy of Sciences of the USSR, The DNC, The POI. Dynamic Processes in the Discrete Geophysical Systems. Collected Book, Vladivostok, $138 \mathrm{c}$.

[9] Ness, N.F., Harrison, J. and Slichter, L.B. (1954) Observation of the natural oscillations of the Earth. Natural oscillations of the earth. Springer-Verlag, New York, 6079.

[10] Pavel, F.G. and Chebotov, S.A. (1987) Some results of studies of high surface deformation. Academy of Sciences of the USSR, The Far East, The POI. The Using of LongBaseline Laser Interferometers in Geophysics. Vladivostok, $75 \mathrm{p}$.

[11] Antonov, L.M. and Savina, N.G. (1987) Analysis of lowfrequency oscillations of the Earth. Recorded by Laser Deformograph.

[12] Davydov, A., Dolgikh, G.I., Kopvillem, W.H. and Zapolskii, A.M. (1987) Study the background of natural oscillations of the Earth. Part II.

[13] Manko, V.I. (2013) Physical Institute RAS. Report of a workshop of theoretical physics under the direction of A. A. Rukhadze. GPI. 